\title{
20C, a bibenzyl compound isolated from Gastrodia elata, protects PC12 cells against rotenone-induced apoptosis via activation of the Nrf2/ARE/HO-1 signaling pathway
}

\author{
Ju-yang HUANG ${ }^{1}$, Yu-he YUAN ${ }^{1}$, Jia-qing YAN ${ }^{1}$, Ya-nan WANG ${ }^{1}$, Shi-feng $\mathrm{CHU}^{2}$, Cheng-gen ZHU ${ }^{1}$, Qing-lan GUO ${ }^{1}$, \\ Jian-gong $\mathrm{SHI}^{1}$, Nai-hong $\mathrm{CHEN}^{1,}$ * \\ ${ }^{1}$ State Key Laboratory of Bioactive Substances and Functions of Natural Medicines, Institute of Materia Medica, Neuroscience Center, \\ Chinese Academy of Medical Sciences and Peking Union Medical College, Beijing 100050, China; ${ }^{2}$ College of Pharmacy, Hunan \\ University of Chinese Medicine, Changsha 410000, China
}

\begin{abstract}
Aim: Our preliminary study shows that a bibenzyl compound isolated from Gastrodia elata, 2-[4-hydroxy-3-(4-hydroxybenzyl)benzyl]-4(4-hydroxybenzyl)phenol (designated 20C), protects PC12 cells against $\mathrm{H}_{2} \mathrm{O}_{2}$-induced injury. In this study we investigated whether $20 \mathrm{C}$ exerted neuroprotective action in a cell model of Parkinson's disease.

Methods: A cell model of Parkinson's disease was established in PC12 cells by exposure to rotenone ( $4 \mu \mathrm{mol} / \mathrm{L})$ for $48 \mathrm{~h}$. Cell viability and apoptosis were assessed, and intracellular ROS level and the mitochondrial membrane potential (MMP) were detected. The expression of apoptosis-related proteins Bax, Bcl-2, cytochrome c, cleaved caspase-3, and oxidative stress-related proteins Nrf2, HO-1 and NQ01 were examined using Western blotting. The mRNA levels of HO-1 and NQO1 were determined with RT-PCR. The nuclear translocation of Nrf2 was observed with immunofluorescence staining.

Results: Treatment with rotenone significantly increased the number of apoptotic cells, accompanied by marked increases in the Bax/ $\mathrm{Bcl}-2$ ratio, cytochrome $\mathrm{c}$ release and caspase- 3 activation. Rotenone also increased ROS accumulation, reduced MMP, and increased the nuclear translocation of Nrf2 as well as the mRNA and protein levels of the Nrf2 downstream target genes HO-1 and NQO1 in PC12 cells. Co-treatment with $20 \mathrm{C}(0.01-1 \mu \mathrm{mol} / \mathrm{L})$ dose-dependently attenuated rotenone-induced apoptosis and oxidative stress in PC12 cells. Nrf2 knockdown by siRNA partially reversed the protective effects of $20 \mathrm{C}$ in rotenone-treated PC12 cells.
\end{abstract}

Conclusion: The bibenzyl compound $20 \mathrm{C}$ protects PC12 cells from rotenone-induced apoptosis, at least in part, via activation of the Nrf2/ARE/HO-1 signaling pathway.

Keywords: bibenzyl compound; Gastrodia elata; rotenone; apoptosis; oxidative stress; Nrf2; neuroprotection; Parkinson's disease; PC12 cells

Acta Pharmacologica Sinica (2016) 37: 731-740; doi: 10.1038/aps.2015.154; published online 16 May 2016

\section{Introduction}

Parkinson's disease (PD) is the second most common agerelated neurodegenerative disorder after Alzheimer's disease $^{[1]}$. Neuropathologically, it is characterized by the progressive loss of dopaminergic neurons and the associated depletion of postsynaptic dopamine within the striatum ${ }^{[2]}$. However, the molecular mechanism underlying dopaminergic neuron death is still not completely understood. One of the major hypotheses posits that mitochondrial dysfunction and

\footnotetext{
* To whom correspondence should be addressed.

E-mail chennh@imm.ac.cn

Received 2015-08-26 Accepted 2015-12-29
}

the subsequent oxidative stress are the main contributors to neuronal cell death ${ }^{[3,4]}$. The overproduction of reactive oxygen species (ROS) in response to oxidative stress induces the peroxidation of lipids and proteins, the oxidation of nucleic acids, and DNA breakdown, thus promoting further cell damage ${ }^{[5]}$.

Rotenone is one of the most widely used pesticides. As a neurotoxin and mitochondrial complex I inhibitor, rotenone has been shown to enhance the formation of intracellular ROS and lead to mitochondrial dysfunction, culminating in apoptotic cell death and is used to create models of PD in vivo and in vitro ${ }^{[6]}$. Recently, a considerable number of reports have shown that different exogenous herbal antioxidants attenuate the oxidative damage caused by rotenone, which emphasizes 
that these promising herbal drugs should be explored as PD therapies ${ }^{[]]}$.

Gastrodia elata (Tianma, GE) is a commonly used traditional Chinese medicine with numerous therapeutic applications, such as for treating vertigo and epilepsy ${ }^{[8]}$. A number of studies suggest that extracts from GE exert antioxidant activity ${ }^{[9,10]}$. 20C is a novel bibenzyl compound isolated from Gastrodia elata. Our previous data indicated that $20 \mathrm{C}$ could protect $\mathrm{PC} 12$ cells against $\mathrm{H}_{2} \mathrm{O}_{2}$-induced injury (data not published). It has not been determined if 20C could inhibit the neurotoxicity of rotenone.

The transcription factor nuclear factor erythroid 2-related factor $2(\mathrm{Nrf} 2)$ is a key protein that regulates the redox state of cells in response to oxidative stress ${ }^{[11]}$. Under physiological, unstressed conditions, Nrf2 is anchored by the Kelchlike ECH-associated protein 1 (Keap1) in the cytoplasm and degraded by the ubiquitin proteasome pathway ${ }^{[12]}$. Once cells encounter an oxidative insult, Nrf2 is released from Keap1 and translocates into the nucleus where it binds to the antioxidant response element (ARE) to activate the transcription of a battery of antioxidant and cytoprotective genes ${ }^{[13]}$. The antioxidant enzymes include hemeoxygenase-1 (HO-1), nicotinamide adenine dinucleotide phosphate: quinine oxidoreductase- 1 (NQO1), superoxide dismutase, and glutathione peroxidase ${ }^{[14]}$.

The Nrf2/ARE/HO-1 signaling pathway is considered to be a protective molecular mechanism in several pathological processes, particularly in oxidative stress, and this pathway is also involved in several central nervous system diseases, including $\mathrm{PD}^{[15,16]}$. In this study, we investigated the anti-apoptotic effects of 20C on PC12 cells intoxicated by rotenone as well as the role of the Nrf2/ARE/HO-1 signaling pathway in this process.

\section{Materials and methods}

\section{Chemicals and reagents}

20C was provided by the Department of Chemosynthesis, Institute of Materia Medica, Chinese Academy of Medical Sciences and Peking Union Medical College (Beijing, China). The chemical structure is shown in Figure 1. 20C was dissolved in dimethyl sulfoxide (DMSO), aliquoted and frozen at $-80^{\circ} \mathrm{C}$ until further use. Rotenone was purchased from SigmaAldrich (St Louis, MO, USA) and freshly dissolved in DMSO prior to each experiment. The Annexin V-FITC/PI staining kit was purchased from Beijing Biosea Biotechnology Company (Beijing, China), and JC-1 and dichlorofluorescin diacetate (DCFH-DA) were obtained from the Beyotime Institute of Biotechnology (Beijing, China).

\section{Cell culture and treatment}

PC12 cells, a rat pheochromocytoma cell line, were purchased from the American Type Culture Collection (ATCC, Manas-

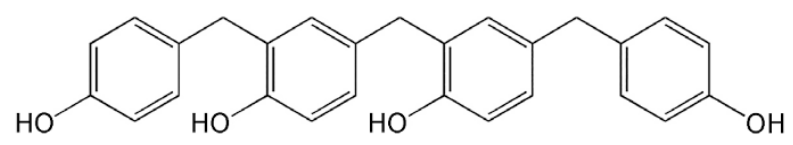

Figure 1. The chemical structure of $20 \mathrm{C}$. sas, VA, USA). The cells were maintained in Dulbecco's Modified Eagle's Medium (DMEM) supplemented with 5\% fetal bovine serum, $10 \%$ heat-inactivated horse serum, 100 $\mathrm{U} / \mathrm{mL}$ penicillin, $100 \mu \mathrm{g} / \mathrm{mL}$ streptomycin, and $2 \mathrm{mmol} / \mathrm{L}$ L-glutamine (Invitrogen, Grand Island, NY, USA). The cells were maintained at $37^{\circ} \mathrm{C}$ in a humidified atmosphere with $5 \%$ $\mathrm{CO}_{2}$ and $95 \%$ air. The media were replaced every two or three days, and the cells were passaged twice per week. The cells were plated in Poly-L-lysine (PLL)-coated plates at an appropriate density, according to the requirements of each experiment. After a 24-h subculture, the cells were switched to fresh medium for treatment. The attached cells were then exposed to $4 \mathrm{\mu mol} / \mathrm{L}$ rotenone in the presence or absence of $0.01,0.1$, $1 \mu \mathrm{mol} / \mathrm{L} 20 \mathrm{C}$ (added immediately before rotenone). For the viability assay and morphological observations, the cells were seeded in 96-well culture plates at a density of $5 \times 10^{4}$ cells $/ \mathrm{mL}$. For immunocytochemical staining and the ROS and mitochondrial membrane potential measurements, the cells were plated on 24-well culture plates at a density of $3 \times 10^{5}$ cells $/ \mathrm{mL}$. For the other experiments, the cells were plated on 6-well culture plates at a density of $3 \times 10^{5}$ cells $/ \mathrm{mL}$. For the MTT (Methyl Thiazolyl Tetrazolium) assay, the cells were treated with $20 \mathrm{C}$ and rotenone for $48 \mathrm{~h}$; for the other experiments, the cells were treated with $20 \mathrm{C}$ and rotenone for $24 \mathrm{~h}$.

\section{Morphological observations and cell viability}

The morphological changes in the different groups of PC12 cells were observed using a phase-contrast microscope (Olympus, Tokyo, Japan). Cell viability was assessed by the MTT assay. The cells were incubated with $10 \mu \mathrm{L}$ of a $5 \mathrm{mg} / \mathrm{mL}$ MTT solution (Sigma-Aldrich) for $4 \mathrm{~h}$ at $37^{\circ} \mathrm{C}$, and then the media were carefully removed. The formazan crystals were dissolved in $100 \mu \mathrm{L}$ of DMSO and the optical density (OD) was measured using a Microplate Reader (Thermo, Waltham, MA, USA) at a wavelength of $570 \mathrm{~nm}$. The cell viability was expressed as a percentage of the OD value of the control cultures.

\section{Analysis of apoptosis by flow cytometry}

The apoptosis rate was measured by flow cytometry, as previously reported ${ }^{[17]}$. After exposure to the indicated doses of rotenone or rotenone plus 20C, the cells were harvested by centrifugation $(800 \times g, 5 \mathrm{~min})$ and washed with ice-cold phosphate-buffered saline (PBS) three times. The apoptosis rate was detected by flow cytometry using an Annexin V-FITC/PI apoptosis detection kit, as previously described ${ }^{[18]}$.

\section{Intracellular ROS measurement}

The intracellular ROS levels were examined using DCFH-DA, and assayed according to the manufacturer's instructions. DCFH-DA, which is oxidized to the fluorescent product DCF in the presence of ROS, was used to measure the relative cellular peroxide levels. Briefly, the cells were washed once with serum-free medium and incubated with $10 \mu \mathrm{mol} / \mathrm{L}$ DCFH-DA for $20 \mathrm{~min}$ at $37^{\circ} \mathrm{C}$ in the dark. Then, the cells were washed with serum-free medium three times and visualized using a fluorescent microscope (Nikon Eclipse Ti-U, Tokyo, Japan). 
The cells were located under bright-field optics and then scanned with the laser (488 nm for excitation and $525 \mathrm{~nm}$ for emission). The fluorescent density was analyzed by ImagePro Plus software version 6.0 (Media Cybemetics, Bethesda, MD, USA).

\section{Measurement of the mitochondrial membrane potential (MMP)}

The changes in the inner MMP were determined by incubating the cells with $10 \mu \mathrm{g} / \mathrm{mL}$ JC-1, a cationic dye used to measure the change in the inner MMP at $37^{\circ} \mathrm{C}$. JC- 1 accumulates in the mitochondria, forming red fluorescent aggregates at high membrane potential; however, at low membrane potential, JC-1 is mainly in the form of green fluorescent monomers. After a 20-min incubation, the cells were rinsed with PBS three times and immediately submitted to fluorescence microscopy analysis. The JC-1-loaded cells were excited at $488 \mathrm{~nm}$, and the emission was detected at $590 \mathrm{~nm}$ (JC-1 aggregates) and 525 nm (JC-1 monomers). Mitochondrial depolarization was indicated by an increase in the green/red fluorescence ratio, which was calculated with Image-Pro Plus software version 6.0.

\section{Western blotting}

The PC12 cells were harvested and lysed in lysis buffer (50 mmol/L Tris-HCl, pH 7.5, $150 \mathrm{~mol} / \mathrm{L} \mathrm{NaCl}, 1$ \% NP-40, and 1 $\mathrm{mol} / \mathrm{L}$ EDTA) for $30 \mathrm{~min}$ in the presence of a protease inhibitor cocktail (Sigma-Aldrich) and phosphatase inhibitors (Thermo) on ice. The lysate was centrifuged for $30 \mathrm{~min}$ at 12000 revolutions/min, and the supernatant was collected and boiled for $10 \mathrm{~min}$ at $100^{\circ} \mathrm{C}$. The nuclear and cytoplasmic proteins were extracted from the cells with the Nuclear-Cytosol Extraction Kit (Applygen Technologies Inc, Beijing, China). The cytoplasmic proteins and mitochondrial proteins were extracted from the cells with the Cell Mitochondria Isolation Kit (Beyotime Institute of Biotechnology, Beijing, China). The protein concentrations were determined using the bicinchoninic acid protein assay (Applygen Technologies Inc). Twenty micrograms of protein from each sample were separated by SDS-PAGE and then transferred to a PVDF membrane (Millipore, Boston, MA, USA). The membrane was blocked with 3\% BSA (SigmaAldrich) and incubated with the following primary antibodies overnight at $4^{\circ} \mathrm{C}$ : anti-Bcl-2, anti-Bax, anti-cytochrome $\mathrm{C}$, anticaspase-3, anti-Nrf2, anti-HO-1, anti-NQO1 (1:500 dilutions; all from Santa Cruz Biotechnology, Dallas, TX, USA), antiPCNA (1:1000 dilution, Cell Signaling Technology, Danvers, MA, USA), and anti- $\beta$-actin (1:5000 dilution, Sigma-Aldrich). The blots were incubated with horseradish peroxidase (HRP)conjugated secondary antibodies (1:5000 dilution, all from KPL, Gaithersburg, MD, USA) for $2 \mathrm{~h}$ at room temperature, and the bands were detected with the enhanced chemiluminescence plus detection system (ImageQuant LAS4000mini, GE, Fairfield, CT, USA). The signals from specific protein brands were quantified using the Gel-Pro Analyzer software (Media Cybernetics).

\section{Immunofluorescence staining}

The PC12 cells were grown on PLL-coated $(0.1 \mathrm{mg} / \mathrm{mL}) \mathrm{Lab}-$
Tek chambered cover glasses (Nunc, IL, USA). The cell culture media was removed and the cells were fixed in $4 \%$ paraformaldehyde for $20 \mathrm{~min}$ at room temperature and washed three times with PBS. The cells were permeabilized in $0.1 \%$ TritonX-100 (Sigma-Aldrich) for $10 \mathrm{~min}$ and blocked in 3\% goat serum for $1 \mathrm{~h}$ at room temperature.

After an overnight incubation with the anti-Nrf2 antibody at $4{ }^{\circ} \mathrm{C}$, the cells were washed three times with PBS and then incubated with secondary antibody (AlexaFluor 546-conjugated goat anti-rabbit antibody, 1:200 dilution, Invitrogen, New York, USA) for $1 \mathrm{~h}$ at room temperature. Afterwards, the cells were washed three times with PBS and stained with Hoechst for $10 \mathrm{~min}$ at room temperature. The images were acquired with a laser scanning confocal microscope fluorescence microscope (Leica TCS SP2, Solms, Germany).

\section{Reverse transcription polymerase chain reaction}

The total RNA was extracted from the PC12 cells in the presence or absence of 20C. One microgram of RNA from each sample was reversed transcribed to cDNA using the reverse transcription kit (Invitrogen) according to the manufacturer's instructions, and amplified by polymerase chain reaction (PCR) with primers specific for $\mathrm{HO}-1$ (forward primer: 5'-ATGGAGCGCCCACAGTCGAC-3', reverse primer: 5'-GGTAGCGGGTATATGCGTGGG-3'), NQO1 (forward primer: 5'-ATGGCGGTGAGAAGAGCCCTGC-3', reverse primer: 5'-ACCCTTGTCATACATGGTGGC-3'), and GAPDH (forward primer: 5'-CATCACCATCTTCCAGGAGCG-3', reverse primer: 5'-TGACCTTGCCCACAGCCTTG-3'). PCR was performed using the following parameters: $95^{\circ} \mathrm{C}$ for 5 min; then, for HO-1: $95^{\circ} \mathrm{C}$ for $30 \mathrm{~s}, 60^{\circ} \mathrm{C}$ for $30 \mathrm{~s}$, and $72^{\circ} \mathrm{C}$ for $30 \mathrm{~s}$; for NQO1: $95^{\circ} \mathrm{C}$ for $30 \mathrm{~s}, 55^{\circ} \mathrm{C}$ for $30 \mathrm{~s}$, and $72^{\circ} \mathrm{C}$ for $30 \mathrm{~s}$; and for GAPDH: $95^{\circ} \mathrm{C}$ for $30 \mathrm{~s}, 60^{\circ} \mathrm{C}$ for $30 \mathrm{~s}$, and $72^{\circ} \mathrm{C}$ for 30 s. After 30 cycles of amplification, a final extension step was performed at $72{ }^{\circ} \mathrm{C}$ for $10 \mathrm{~min}$. The amplified cDNAs were separated by electrophoresis on a $1 \%$ agarose gel, stained with ethidium bromide, and visualized using an electrophoresis gel imaging analysis system and Quantity One software (Bio-Rad, Hercules, CA, USA).

\section{Nrf2-siRNA knockdown}

The PC12 cells were grown to $60 \%-80 \%$ confluence in culture media. The cells were transfected with Nrf2-siRNA or controlsiRNA using an siRNA transfection reagent (Santa Cruz Biotechnology), according to the manufacturer's protocol. The final concentration of the siRNA was $60 \mathrm{nmol} / \mathrm{L}$. The knockdown efficiency was validated by Western blotting. To confirm the specificity of the interference, a nontargeting siRNA was used as a negative control. After $24 \mathrm{~h}$ of transfection, the transfection solution was removed and the cells were rinsed with PBS and treated with rotenone in the presence or absence of 20C. The cells were collected for the cell viability assay.

\section{Statistical analysis}

The data were expressed as the means \pm SD. Significant differences between the experimental and control groups were 
assessed by Student's t-test (Figure 6A) and one-way ANOVA using GraphPad Prism 5 software (La Jolla, CA, USA). $P<0.05$ was considered statistically significant.

\section{Results}

20C protected PC12 cells against the rotenone-induced apoptosis

As shown in Figure 2A, the rotenone-treated PC12 cells exhibited morphological alterations that are normally associated with apoptosis, such as cell shrinkage and membrane blebbing. The 20C treatment alleviated these morphological features of damaged cells. Using the MTT assay, we showed that rotenone induced remarkable cytotoxicity in the PC12 cells, with only $66.2 \%$ of the cells remaining viable (Figure 2D). When the cells were treated with $0.01,0.1$ and $1 \mu \mathrm{mol} / \mathrm{L}$ 20C, the cell viability was restored to $72.4 \%, 78.2 \%$ and $83.3 \%$, respectively.

The anti-apoptosis effect of 20C in the PC12 was further studied using the Annexin V-FITC/PI assay. As shown in Figure 2B and $\mathrm{C}$, the percentage of $\mathrm{PC} 12$ cells undergoing early apoptosis following rotenone treatment was significantly increased $(P<0.01)$. Moreover, the 0.1 and $1 \mu \mathrm{mol} / \mathrm{L}$ doses of $20 \mathrm{C}$ reduced the rotenone-induced apoptosis $(P<0.05, P<0.05)$.

$20 \mathrm{C}$ suppressed the pro-apoptotic effect of rotenone by inhibiting the increase in the $\mathrm{Bax} / \mathrm{Bcl}-2$ ratio, cytochrome $c$ release, and caspase- 3 cleavage

In this study, we demonstrated that 20C (0.01, 0.1 and 1 $\mu \mathrm{mol} / \mathrm{L}$ ) inhibited the rotenone-induced up-regulation of Bax and down-regulation of Bcl-2, thus decreasing the Bax/Bcl-2 ratio, as shown in Figure $3 \mathrm{~A}(P<0.01)$. Additionally, the cytoplasmic cytochrome $C$ release was decreased in the cells that were treated with various concentrations of $20 \mathrm{C}(0.01,0.1$ and $1 \mathrm{mmol} / \mathrm{L} ; \mathrm{P}<0.01)$ compared with the rotenone-treated group (Figure 3B). Furthermore, we assessed the caspase-3 cleavage by determining the concentration of cleaved caspase-3 (17
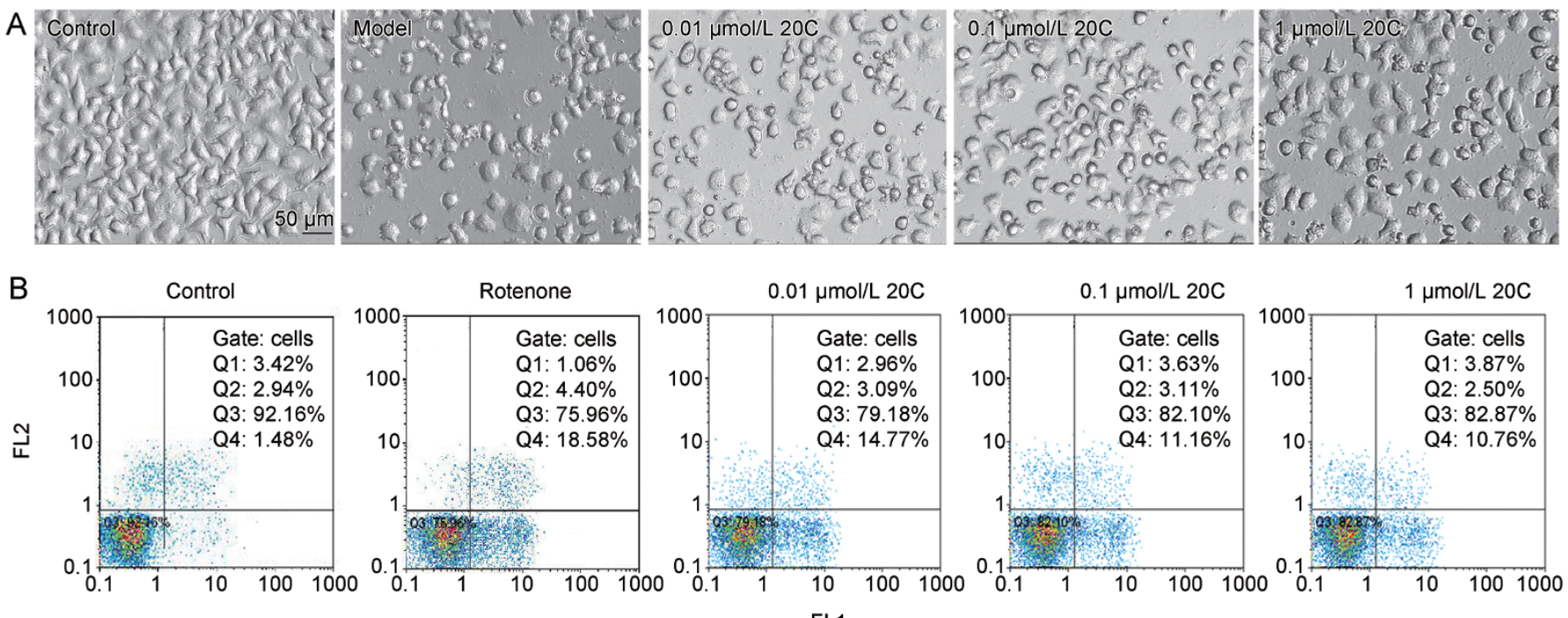

C
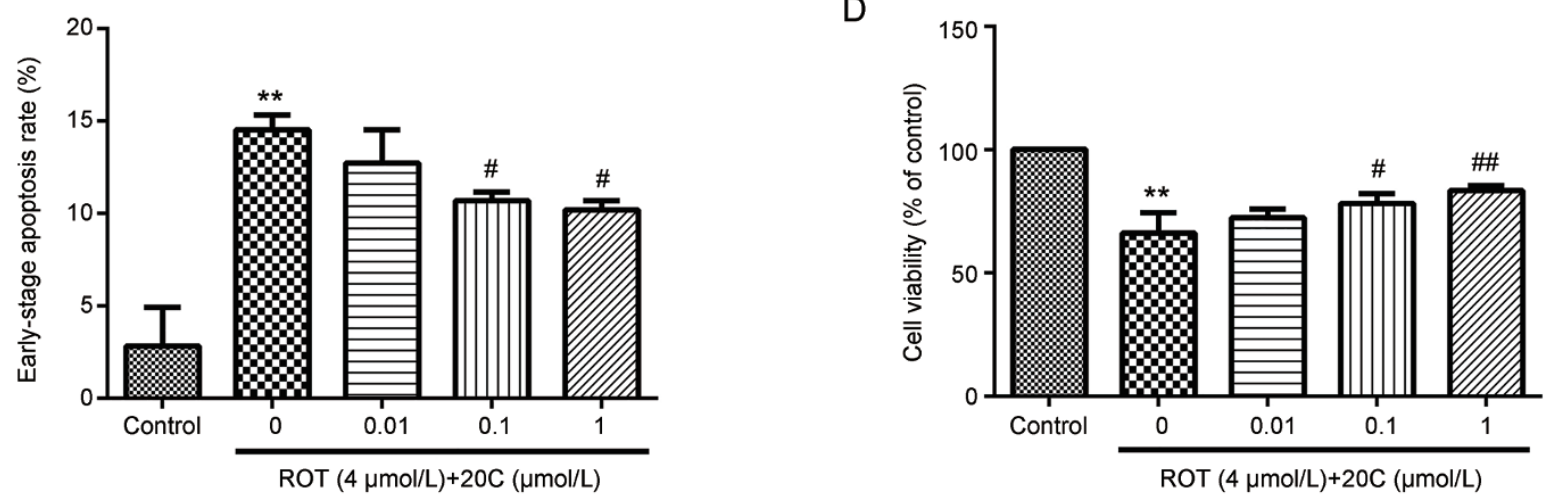

Figure 2. Effects of $20 \mathrm{C}$ on rotenone-induced apoptosis. (A) Phase-contrast micrographs of PC12 cells exposed to $4 \mu \mathrm{mol} / \mathrm{L}$ rotenone for $48 \mathrm{~h}$ in the presence or absence of 20C. (B, C) Flow cytometry analysis of rotenone-induced apoptosis in PC12 cells. (B) The graphs of apoptosis were obtained from the flow cytometry analysis. (C) Quantitative analysis of the ratio of early apoptotic cells. (D) Cell viability was measured by the MTT assay. The absorbance of the control cells was set as $100 \%$. The data were obtained from three independent experiments. ${ }^{* *} P<0.01$ vs the control group, ${ }^{\#} P<0.05,{ }^{\#} P<0.01$ vs the model group $(n=3)$. ROT, rotenone. 
A
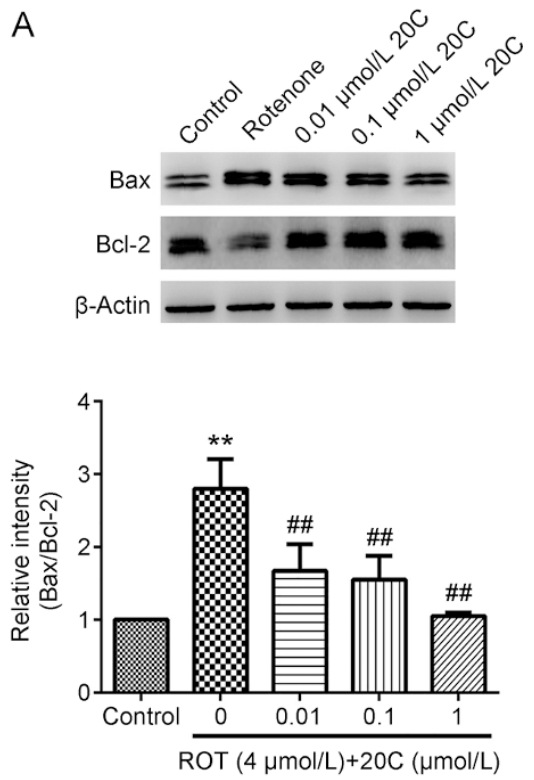

B
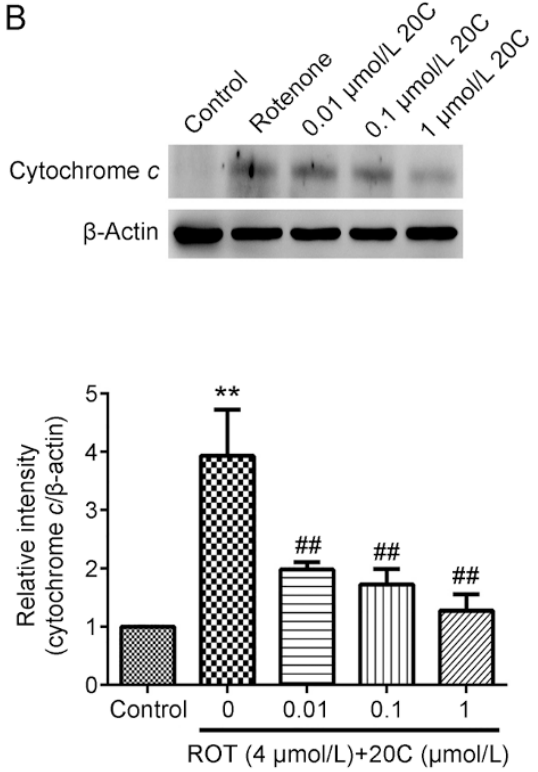

C
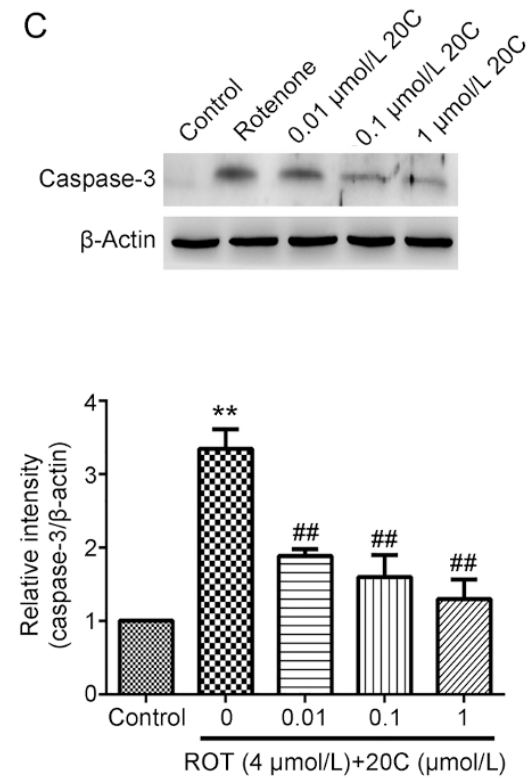

Figure 3. Effects of $20 \mathrm{C}$ on the expression of apoptosis-related proteins. (A) Western blotting analysis of the levels of the Bax and Bcl-2 proteins in PC12 cells exposed to rotenone in the presence or absence of various concentrations of 20C. (B) Western blotting analysis of the levels of the cytoplasmic cytochrome C protein in PC12 cells exposed to rotenone in the presence or absence of various concentrations of 20C. (C) Western blotting analysis of the levels of the cleaved caspase-3 protein in PC12 cells exposed to rotenone in the presence or absence of various concentrations of $20 \mathrm{C}$. ${ }^{* *} P<0.01$ vs the control group, ${ }^{\# \#} P<0.01$ vs the model group $(n=3)$.

$\mathrm{kD}$ ). As shown in Figure 3C, the rotenone-induced increase in cleaved caspase- 3 was reversed by co-treatment with $20 \mathrm{C}$ at doses of $0.01,0.1$ and $1 \mu \mathrm{mol} / \mathrm{L}(P<0.01)$.

20C suppressed the accumulation of intracellular ROS and the collapse of the mitochondrial membrane potential

To further study the mechanisms underlying the protective effect of 20C, the intracellular ROS levels were determined using DCFH-DA and fluorescence microscopy. As shown in Figure 4A, normal PC12 cells exhibited weak green fluorescence, and the green fluorescence was remarkably enhanced following rotenone exposure $(P<0.01)$. In the $20 \mathrm{C}$ treatment group, the intensity of the green fluorescence was significantly reduced (Figure $4 \mathrm{C}, P<0.01$ ).

The MMP was identified using the mitochondria-specific fluorescent dye JC-1. Normal PC12 cells stained with the JC-1 dye emitted a mitochondrial orange-red fluorescence, with a small amount of green fluorescence, as shown in Figure $4 \mathrm{~B}$. These JC-1 aggregates within the normal mitochondria were dispersed into the monomeric form (green fluorescence) upon addition of rotenone to the culture medium. After treatment with $0.01,0.1$ and $1 \mu \mathrm{mol} / \mathrm{L} \mathrm{20C}$, the ratio of green/red fluorescence was significantly decreased (Figure 4D, $P<0.05$, $P<0.01, P<0.01)$.

$20 \mathrm{C}$ promoted Nrf2 translocation from the cytoplasm to the nucleus and the expression of its downstream factors

To gain further insights into the molecular mechanisms underlying the anti-apoptosis effect of 20C in PC12 cells, the transcription factor Nrf2 was examined as a potential upstream regulator of the cellular antioxidant system. The well-established, classical activation pattern of Nrf2 involves its translocation from the cytoplasm to the nucleus. Therefore, we first investigated the nuclear accumulation of Nrf2 protein in the cells treated with 20C. The results obtained from the Western blotting analysis showed that treatment with 0.1 and $1 \mu \mathrm{mol} / \mathrm{L} 20 \mathrm{C}$ resulted in a significant accumulation of Nrf2 in the nucleus $(P<0.05, P<0.01)$ and a decrease in cytoplasmic Nrf2 in a dose-dependent manner (Figure 5A and B, $P<0.01)$. The nuclear translocation of Nrf2 was also confirmed by immunofluorescence. In the control and rotenone-treated groups, Nrf2 was predominantly located in the cytoplasm, whereas Nrf2 translocated from the cytoplasm to the nucleus in the cells treated with $1 \mu \mathrm{mol} / \mathrm{L} \mathrm{20C} \mathrm{(Figure} \mathrm{5C).}$

The above observations showed that 20C promoted Nrf2 nuclear translocation and then activated Nrf2. Thus, we hypothesized that 20C might regulate Nrf2 downstream target genes. Therefore, we investigated the expression of the downstream factors in this pathway. As shown in Figure 5D and 5E, the HO-1 proteins were up-regulated after the $0.1 \mu \mathrm{mol} / \mathrm{L} 20 \mathrm{C}$ treatment $(P<0.05)$, while both the HO-1 and NQO1 proteins were up-regulated after the $1 \mu \mathrm{mol} / \mathrm{L} 20 \mathrm{C}$ treatment $(P<0.01$, $P<0.01)$. Moreover, the mRNA levels were consistent with the changes in the protein levels; $0.1 \mu \mathrm{mol} / \mathrm{L}$ and $1 \mu \mathrm{mol} / \mathrm{L} 20 \mathrm{C}$ enhanced the transcription of the HO-1 $(P<0.05, P<0.05)$ and NQO1 mRNAs $(P<0.01, P<0.05)$ compared with the rotenonetreated group (Figure 5F and 5G). These results indicated that $20 \mathrm{C}$ induced the expression of the mRNAs and proteins of Nrf2 downstream factors by activating the Nrf2 signaling pathway. 

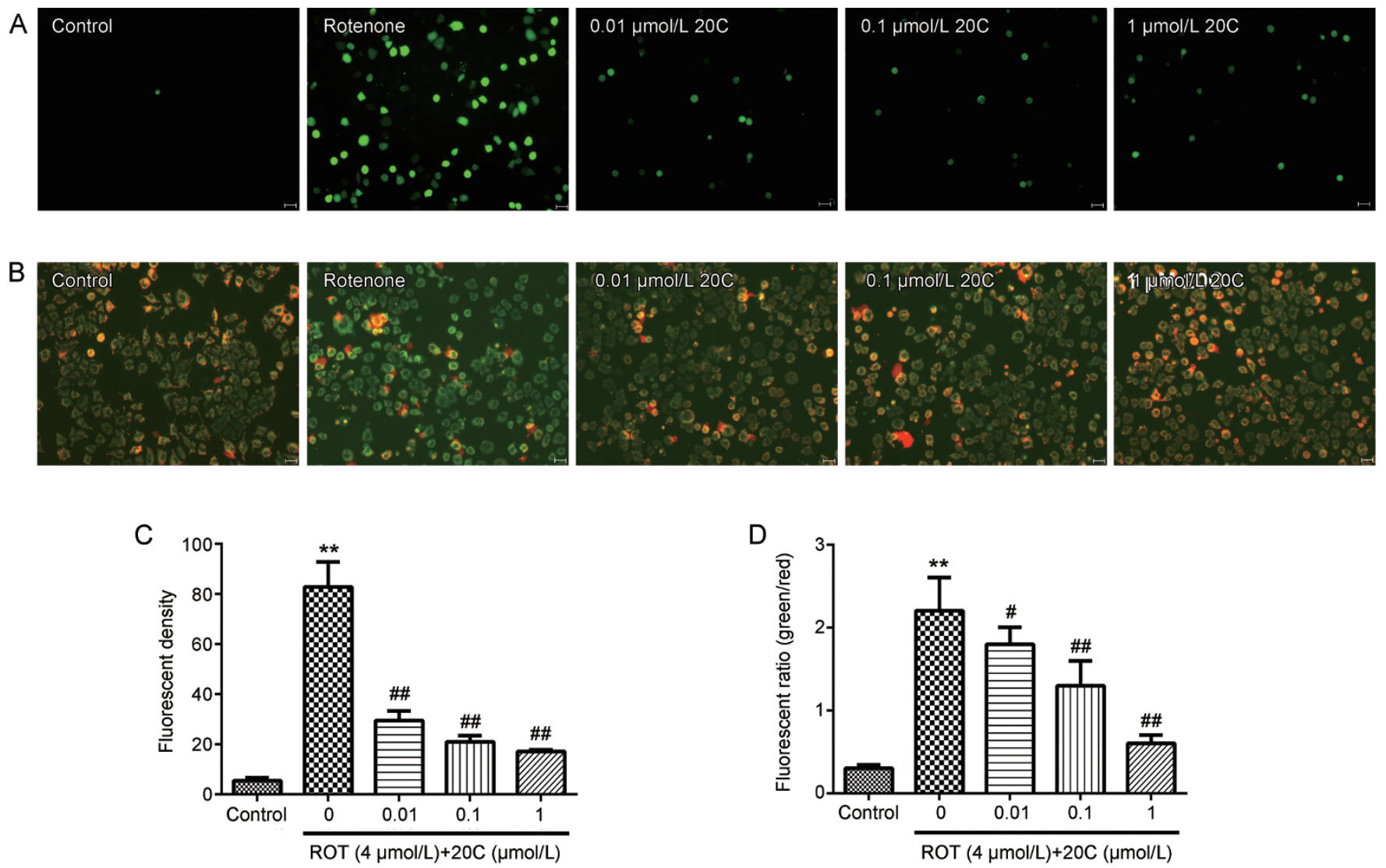

Figure 4. Effects of $20 \mathrm{C}$ on rotenone-induced oxidative stress. (A, B) The ROS levels (A) and MMP (B) of PC12 cells exposed to rotenone in the presence or absence of $20 \mathrm{C}$ were determined using DCFH-DA (A) and JC-1 (B). The scale bar represents $20 \mu \mathrm{m}$. (C, D) Quantitative analysis of the ROS levels (C) and MMP (D). ${ }^{* *} P<0.01$ vs the control group, ${ }^{\#} P<0.05$, ${ }^{\# \#} P<0.01$ vs the model group $(n=3)$.

The protective effect of $20 \mathrm{C}$ is involved the activation of Nrf2

To provide direct evidence for the involvement of Nrf2 activation in 20C-mediated cytoprotection, we transfected PC12 cells with either Nrf2-siRNA or control-siRNA for $24 \mathrm{~h}$, followed by treatment with $4 \mathrm{\mu mol} / \mathrm{L}$ rotenone in the presence or absence of $0.01,0.1,1 \mu \mathrm{mol} / \mathrm{L} 20 \mathrm{C}$ for an additional $48 \mathrm{~h}$. The efficiency of the Nrf2-siRNA was verified by Western blotting. As shown in Figure 6A, the Nrf2-siRNA treatment significantly decreased the level of Nrf2 $(P<0.01)$. We subsequently exposed the Nrf2-siRNA- and control-siRNA-transfected PC12 cells to rotenone with or without 20C. As expected, 0.1 and 1 $\mathrm{mol} / \mathrm{L} 20 \mathrm{C}$ protected the control-siRNA-treated cells against rotenone-induced cell death $(P<0.05, P<0.01)$, but only the $1 \mu \mathrm{mol} / \mathrm{L} 20 \mathrm{C}$ dose exerted a protective effect on the Nrf2siRNA-treated cells (Figure 6B, $P<0.05$ ). Taken together, these results suggested that $20 \mathrm{C}$ partially protected the cells against rotenone-induced death by activating Nrf2.

\section{Discussion}

Accumulating evidence suggests that exposure to environmental toxins, such as pesticides, could be an underlying risk factor for the development of many neurodegenerative diseases, including Parkinson's disease. Rotenone, a pesticide and specific inhibitor of mitochondrial complex I, has consis- tently been reported to be associated with the development of PD in human populations. Several studies have shown that mitochondrial dysfunction, oxidative stress, and cell death are important factors in the pathogenesis of $\mathrm{PD}^{[19]}$. In fact, exposure to rotenone induces critical damage to mitochondria, enhances ROS formation and provokes oxidative damage, resulting in cell death ${ }^{[20]}$. The generation of ROS is an essential metabolic process for maintaining homeostasis. The imbalance between ROS production and the antioxidative stress defense systems can be highly deleterious to the cells. Nrf2 is known to be an important transcription factor that is involved in the cellular antioxidant response, as it binds to antioxidant response elements (ARE) in the genes encoding detoxification enzymes, such as HO-1 and NQO1 ${ }^{[21]}$. Moreover, it has also been reported that $\mathrm{Nrf} 2$ activation provides insights into the treatment of $\mathrm{PD}^{[15]}$. In this study, we demonstrated that 20C protected PC12 cells against rotenone-induced toxicity. The underlying mechanisms were associated with the inhibition of rotenone-induced oxidative stress by activating Nrf2 and its downstream target genes.

PC12 cells, which share many properties with primary sympathetic neurons and chromaffin cell cultures, were used to establish the Parkinsonism cell model ${ }^{[22]}$. Consistent with previous reports, our results showed that rotenone primarily 
A

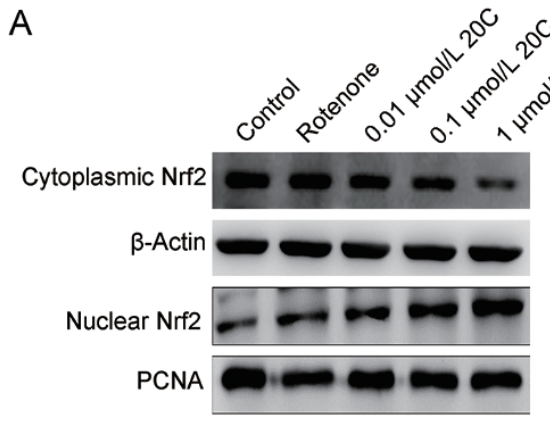

B
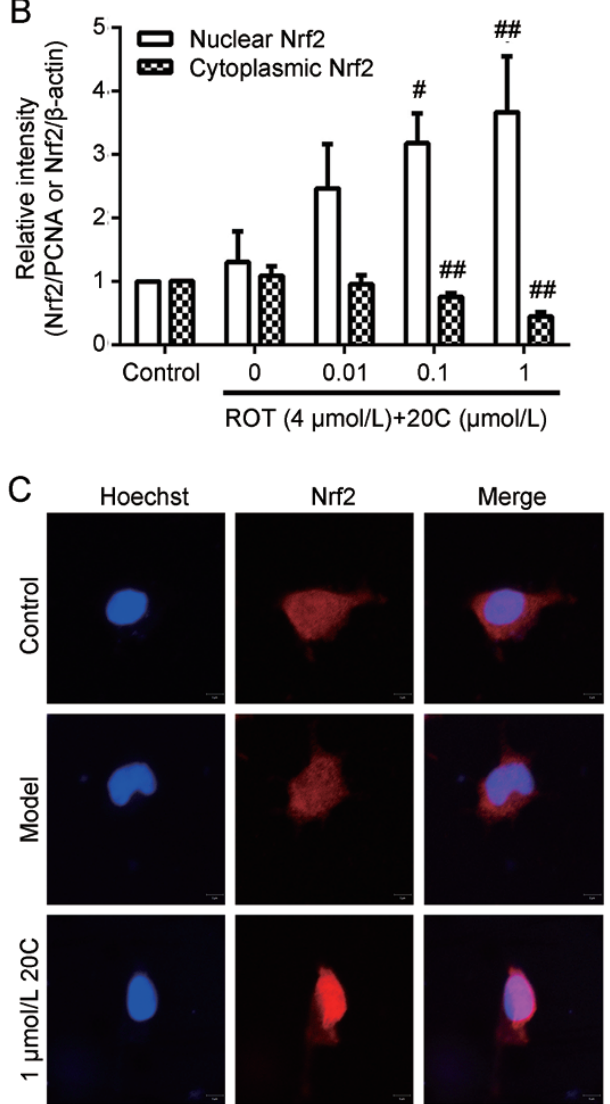

D
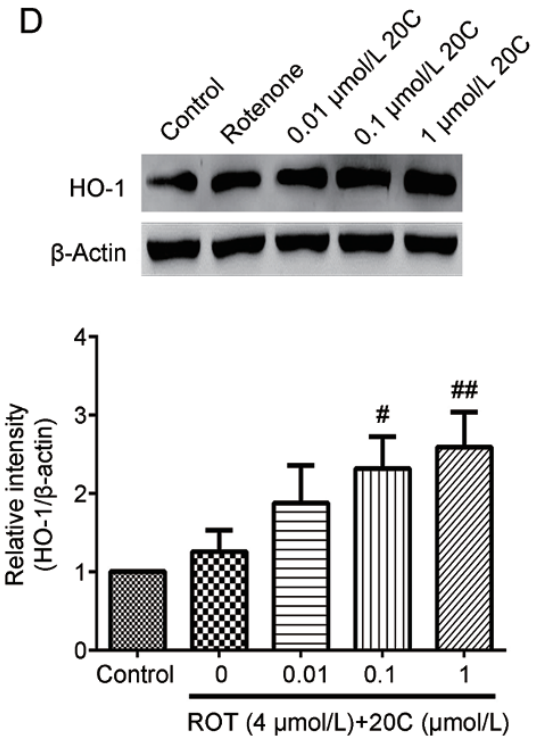

E
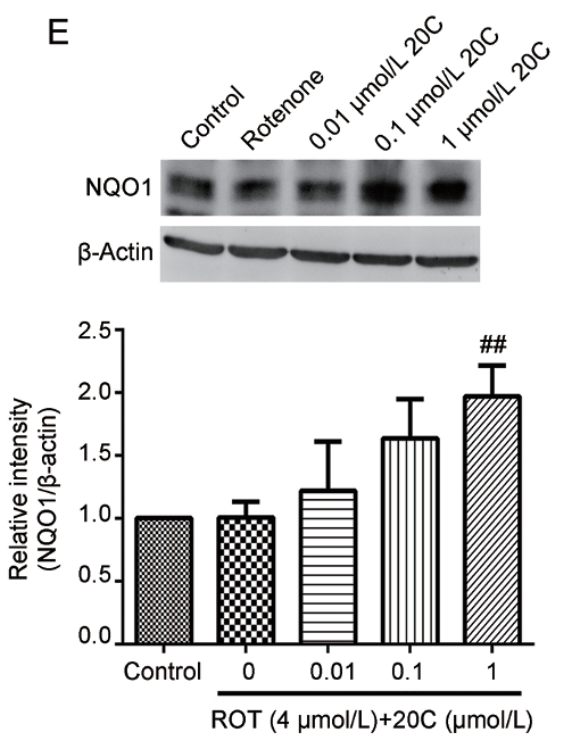

F
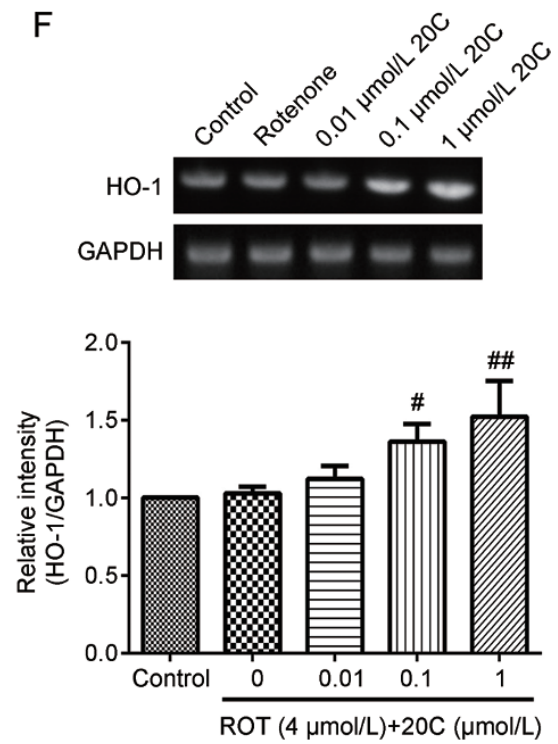

G
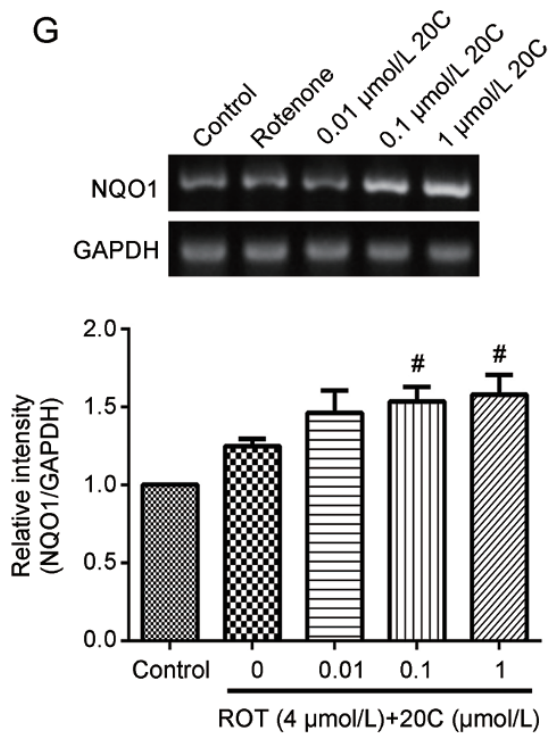

Figure 5. Effects of $20 \mathrm{C}$ on the Nrf2/ARE/HO-1 signaling pathway. (A) Western blotting analysis of the levels of the Nrf2 protein in the cytoplasm and nucleus of PC12 cells exposed to rotenone in the presence or absence of various concentrations of 20C. (B) Quantitative analysis of the density of Nrf2 in the cytoplasm and nucleus. (C) Confocal microscopy images of immunofluorescence with an anti-Nrf2 antibody showed the nuclear translocation of Nrf2 in the 20C-treated PC12 cells. The scale bar represents $10 \mu \mathrm{m}$. (D, E) Western blotting analysis of the levels of the HO-1 and NQ01 proteins in PC12 cells exposed to rotenone in the presence or absence of 20C. (F, G) RT-PCR analysis of the mRNA levels of HO-1 and NQ01 in PC12 cells exposed to rotenone in the presence or absence of $20 \mathrm{C}$. ${ }^{\#} P<0.05$, ${ }^{\# \#} P<0.01$ vs the model group $(n=3)$.

induced PC12 cell death through apoptosis ${ }^{[23]}$. 20C exerted protective effects against rotenone-induced neurotoxicity, as evidenced by the findings that the rotenone-induced morphological alterations, decreased cell viability and elevated apoptosis were ameliorated by 20C. These results demonstrated that 20C protected PC12 cells against rotenone-induced apoptosis.

The Bcl-2 family of proteins is an important endogenous regulator of cellular activity in response to a variety of physiological and pathological insults, including the mitochondrial pathway of apoptosis. The Bcl-2 family is composed of antiapoptotic proteins such as Bcl-2 and pro-apoptotic proteins, including Bax. During apoptosis, cytochrome $\mathrm{C}$ is translocated from the mitochondrial membrane to the cytosol, where it is required for the activation of caspase-3. Overexpression of Bcl-2 has been shown to prevent the translocation of cytochrome $\mathrm{C}$, thereby blocking the apoptotic process. Caspase-3 is a critical executioner of apoptosis and one of the critical enzymes in rotenone-induced apoptosis in neuronal cells ${ }^{[24]}$. In the present study, $20 \mathrm{C}$ regulated the levels of Bcl-2 family 
A
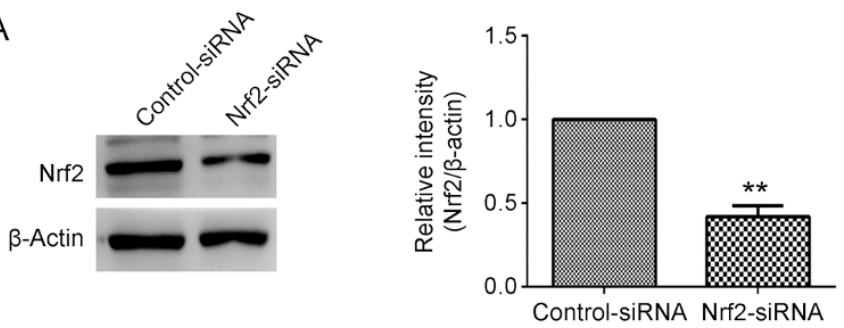

B

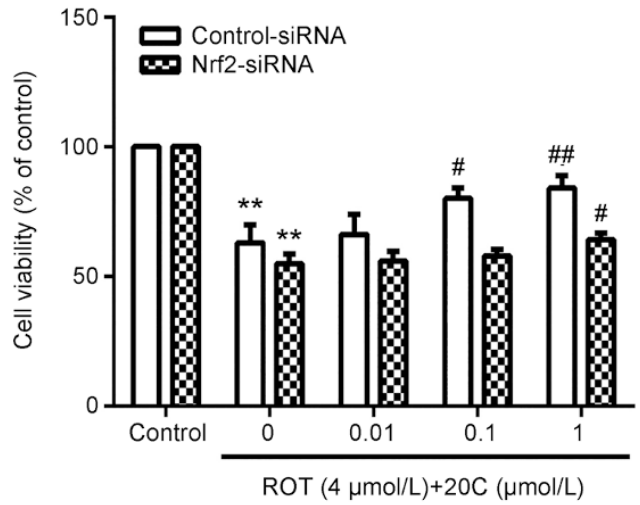

Figure 6. Nrf2 activation is involved in the protective effect of $20 \mathrm{C}$. (A) Transient transfection of PC12 cells with Nrf2-siRNA inhibited the expression of the Nrf2 protein. Western blotting analysis of the levels of the Nrf2 protein in PC12 cells $24 \mathrm{~h}$ after transfection. (B) The PC12 cells were transfected with the control-siRNA or Nrf2-siRNA for $24 \mathrm{~h}$, and then subjected to $4 \mu \mathrm{mol} / \mathrm{L}$ rotenone treatment for an additional $48 \mathrm{~h}$ in the presence or absence of $20 \mathrm{C}$. Cell survival was assessed by the MTT assay. ${ }^{* *} P<0.01$ vs the control group, ${ }^{\#} P<0.05$, ${ }^{\# \#} P<0.01$ vs the model group $(n=3)$.

proteins, and then inhibited cytochrome $\mathrm{C}$ release and the production of active caspase-3 $(17 \mathrm{kD})$ in the rotenone-treated PC12 cells. In summary, 20C prevented rotenone-induced apoptosis, as determined by the $\mathrm{Bax} / \mathrm{Bcl}-2$ ratio, cytochrome $\mathrm{C}$ release and caspase- 3 cleavage.

Mitochondria are involved in cell survival and play a central role in apoptosis through the control of cellular energy metabolism, the generation of ROS, and the release of apoptotic factors into the cytoplasm. Previous evidence showed that ROS was involved in the apoptotic mechanism of rotenone-induced neurotoxicity ${ }^{[25]}$. In addition to the intracellular ROS levels, the changes in MMP during apoptosis are also consistent with mitochondrial dysfunction ${ }^{[26]}$. Our data showed that rotenone significantly aggravated MMP collapse and induced the over production of intracellular ROS. However, 20C could attenuate the rotenone-induced MMP collapse and intracellular ROS overload, which indicated that $20 \mathrm{C}$ contributed to the recovery of mitochondrial function.

Considering the positive effect of $20 \mathrm{C}$ on intracellular ROS production and $\mathrm{H}_{2} \mathrm{O}_{2}$-induced injury (data unpublished), we speculated that $20 \mathrm{C}$ might participate in the cellular antioxidant response. Nrf2 is an essential mediator of the expression of antioxidant enzymes and stress-inducible proteins. The activation of Nrf2 in cells provides an indirect way to enhance their antioxidant capacity, thereby preventing cellular dysfunction in response to free radicals. Under non-oxidizing conditions, Nrf2 binds to Keap1, forming a complex that remains sequestered in the cytoplasm ${ }^{[27]}$. Following an oxidative insult, Nrf2 dissociates from the Keap1-Nrf2 complex and translocates to the nucleus, binding to ARE and potentiating the cellular response against oxidative stress ${ }^{[28]}$. Among the target genes regulated by Nrf2, both HO-1 and NQO1 participate in the antioxidant response. Numerous evidence has showed that HO-1 plays an important role in neuroprotective function against oxidative injury ${ }^{[2]}$. In addition, the expression of antioxidative enzyme HO- 1 exerts a neuroprotective effect by protecting the dopaminergic neurons ${ }^{[30]}$. NQO1 is a cytoplasmic two electron reductase that catalyzes the reduction of a wide range of substrates, including quinones, quinone-imines, and nitro-compounds ${ }^{[3]]}$. Thus, the up-regulation of HO-1 and NQO1 in response to oxidative stresses provides an effective endogenous antioxidant defense mechanism in rotenone-induced PC12 cells $^{[32,33]}$. To determine whether the Nrf2/ARE/HO-1 signaling pathway is involved in the neuroprotective effect of $20 \mathrm{C}$, we investigated the changes in the Nrf2/ARE/HO-1 signaling pathway in the 20C-treated cells. The present data showed that $20 \mathrm{C}$ significantly promoted the translocation of Nrf2 from the cytoplasm to the nucleus, which indicated that Nrf2 was activated. Meanwhile, the upregulated mRNA and protein levels of HO-1 and NQO1 in the 20C-treated PC12 cells were associated with Nrf2 activation. Collectively, these results demonstrated that 20C improved the antioxidant capacity of $\mathrm{PC} 12$ cells against rotenone-induced damage as a result of Nrf2/ARE/HO-1 activation.

Because the regulation of antioxidant response involves a number of signaling pathways, we hypothesized that the mechanism of the protective effect of 20C treatment was associated with the downstream pathways activated by Nrf2. RNAi-mediated Nrf2 knockdown diminished the cytoprotective effects of 20C, which strongly suggests that the Nrf2/ HO-1/NQO1 pathway was activated. These results are similar to previous studies demonstrating that Nrf2 plays a key role in protecting cells against oxidative stress ${ }^{[34-36]}$. However, in the MTT assay, $1 \mu \mathrm{mol} / \mathrm{L} 20 \mathrm{C}$ had a beneficial effect against rotenone-induced apoptosis in Nrf2-knockdown PC12 cells, which indicated that, 20C might activate other targets, in addition to Nrf2/ARE/HO-1 signaling pathway, to exert its cytoprotective effect. We have demonstrated that $20 \mathrm{C}$ regulated the levels of the Bcl-2 family proteins, reduced ROS accumulation and stabilized the mitochondrial membrane potential, which suggested that 20C inhibited the mitochondria-dependent cell death pathway. We speculated that in addition to Nrf2/ ARE/HO-1 signaling pathway, 20C might have direct or indirect effects on the mitochondria to inhibit the mitochondriadependent cell death pathway. Further research is required to explore the effect of $20 \mathrm{C}$ on the mitochondria. Taken together, these results demonstrated that 20C protected PC12 cells against rotenone-induced apoptosis, and the mechanism involved the activation of the Nrf2/ARE/HO-1 signaling 
pathway.

In conclusion, our present observations identified a beneficial effect from treatment with 20C, a brand new bibenzyl compound, against rotenone-induced apoptosis in PC12 cells, which operates, at least in part, via the activation of the Nrf2/ ARE/HO-1 signaling pathway. The study provides further evidence of the beneficial effects of $20 \mathrm{C}$ in the prevention of rotenone-induced toxicity in PC12 cells, which suggested that 20C could be a leading candidate for the treatment of Parkinson's disease.

\section{Acknowledgements}

This work was supported by the National Natural Science Foundation of China (№ 81274122, № 81102831, and № $81273629)$, the National Key Sci-Tech Major Special Item (No 2012ZX09301002-004 and No 2012ZX09103101-006), the National High-Tech R\&D Programme (863 Program) (No 2012AA020303), the Program for Changjiang Scholars and Innovative Research Team in University (PCSIRT) (No IRT1007), the Specialized Research Fund for the Doctoral Program of Higher Education of China (№ 20121106130001), Beijing Natural Science Foundation (№ 7131013 and № 7142115), and Beijing Key Laboratory of New Drug Mechanisms and Pharmacological Evaluation Study (№ BZ0150).

\section{Author contribution}

Ju-yang HUANG and Nai-hong CHEN proposed the study concepts; Ju-yang HUANG designed and performed the experiments and performed the data analysis; Yu-he YUAN, Jia-qing YAN, and Shi-feng CHU prepared the manuscript; Ya-nan WANG, Cheng-gen ZHU, Qing-lan GUO, and Jiangong $\mathrm{SHI}$ assisted in preparing the chemical reagents.

\section{References}

1 Dauer W, Przedborski S. Parkinson's Disease: mechanisms and models. Neuron 2003; 39: 889-909.

2 Ozansoy M, Basak AN. The central theme of Parkinson's disease: alpha-synuclein. Mol Neurobiol 2013; 47: 460-5.

3 Schapira AH. Mitochondria in the etiology and pathogenesis of Parkinson's disease. Lancet Neurol 2008; 7: 97-109.

4 Trushina E, Mcmurray CT. Oxidative stress and mitochondrial dysfunction in neurodegenerative diseases. Neuroscience 2007; 145: $1233-48$.

5 Perier C, Bove J, Vila M, Przedborski S. The rotenone model of Parkinson's disease. Trends Neurosci 2003; 26: 345-6.

6 Li N, Ragheb KE, Lawler G, Sturgis J, Rajwa B, Melendez JA, et al. Mitochondrial complex I inhibitor rotenone induces apoptosis through enhancing mitochondrial reactive oxygen species production. J Biol Chem 2003; 278: 8516-25.

7 Girish C, Muralidhara. Propensity of Selaginella delicatula aqueous extract to offset rotenone-induced oxidative dysfunctions and neurotoxicity in Drosophila mela-nogaster: implications for Parkinson's disease. Neurotoxicology 2012; 33: 444-56.

8 Ojemann LM, Nelson WL, Shin DS, Rowe AO, Buchanan RA. Tian ma, an ancient Chinese herb, offers new options for the treatment of epilepsy and other conditions. Epilepsy Behav 2006; 8: 376-83.

9 Shin EJ, Bach JH, Nguyen TT, Nguyen XK, Jung BD, Oh KW, et al. Gastrodia elata bl attenuates methamphetamine-induced dopaminergic toxicity via inhibiting oxidative burdens. Curr Neuropharmacol 2011; 9: 118-21.

10 Liu J, Mori A. Antioxidant and free radical scavenging activities of Gastrodia elata BI. and Uncaria rhynchophylla (Miq.) Jacks. Neuropharmacology 1992; 31: 1287-98.

11 Kobayashi M, Yamamoto M. Nrf2-Keap1 regulation of cellular defense mechanisms against electrophiles and reactive oxygen species. Adv Enzyme Regul 2006; 46: 113-40.

12 Kobayashi A, Kang MI, Okawa H, Ohtsuji M, Zenke Y, Chiba T, et al. Oxidative stress sensor Keap1 functions as an adaptor for Cul3-based E3 ligase to regulate proteasomal degradation of Nrf2. Mol Cell Biol 2004; 24: 7130-9.

13 De Vries HE, Witte M, Hondius D, Rozemuller AJ, Drukarch B, Hoozemans J, et al. Nrf2-induced antioxidant protection: a promising target to counteract ROS-mediated damage in neurodegenerative disease? Free Radic Biol Med 2008; 45: 1375-83.

14 Cho HY, Reddy SP, Debiase A, Yamamoto M, Kleeberger SR. Gene expression profiling of NRF2-mediated protection against oxidative injury. Free Radic Biol Med 2005; 38: 325-43.

15 Barone MC, Sykiotis GP, Bohmann D. Genetic activation of Nrf2 signaling is sufficient to ameliorate neurodegenerative phenotypes in a Drosophila model of Parkinson's disease. Dis Model Mech 2011; 4: 701-7.

16 Tufekci KU, Civi Bayin E, Genc S, Genc K. The Nrf2/ARE pathway: a promising target to counteract mitochondrial dysfunction in Parkinson's disease. Parkinsons Dis 2011; 314082.

17 Nicoletti I, Migliorati G, Pagliacci MC, Grignani F, Riccardi C. A rapid and simple method for measuring thymocyte apoptosis by propidium iodide staining and flow cytometry. J Immunol Methods 1991; 139: 271-9.

18 Song XY, Hu JF, Chu SF, Li ZP, Wu DH, Ji HJ, et al. IMM-H0O4, a novel coumarin derivative compound, protects against amyloid betainduced neurotoxicity through a mitochondrial-dependent pathway. Neuroscience 2013; 242: 28-38.

19 Lin MT, Beal MF. Mitochondrial dysfunction and oxidative stress in neurodegenerative diseases. Nature 2006; 443: 787-95.

20 Betarbet R, Sherer TB, MacKenzie G, Garcia-Osuna M, Panov AV, Greenamyre JT. Chronic systemic pesticide exposure reproduces features of Parkinson's disease. Nat Neurosci 2000; 3: 1301-6.

21 Scapagnini G, Vasto S, Abraham NG, Caruso C, Zella D, Fabio G. Modulation of Nrf2/ARE pathway by food polyphenols: a nutritional neuroprotective strategy for cognitive and neurodegenerative disorders. Mol Neurobiol 2011; 44: 192-201.

22 Greene LA, Tischler AS. Establishment of a noradrenergic clonal line of rat adrenal pheochromocytoma cells which respond to nerve growth factor. Proc Natl Acad Sci U S A 1976; 73: 2424-8.

$23 \mathrm{Li} \mathrm{BY}$, Yuan YH, Hu JF, Zhao Q, Zhang DM, Chen NH. Protective effect of Bu-7, a flavonoid extracted from Clausena lansium, against rotenone injury in PC12 cells. Acta Pharmacol Sin 2011; 32: 1321-6.

24 Pei W, Liou AK, Chen J. Two caspase-mediated apoptotic pathways induced by rotenone toxicity in cortical neuronal cells. FASEB J 2003; 17: 520-2.

25 Tan S, Sagara Y, Liu Y, Maher P, Schubert D. The regulation of reactive oxygen species production during programmed cell death. J Cell Biol 1998; 141: 1423-32.

26 Kim WH, Park WB, Gao B, Jung MH. Critical role of reactive oxygen species and mitochondrial membrane potential in Korean mistletoe lectin-induced apoptosis in human hepatocarcinoma cells. Mol Pharmacol 2004; 66: 1383-96.

27 Velichkova M, Hasson T. Keap1 regulates the oxidation-sensitive shuttling of Nrf2 into and out of the nucleus via a Crm1-dependent 
nuclear export mechanism. Mol Cell Biol 2005; 25: 4501-13.

28 Wakabayashi N, Dinkova-Kostova AT, Holtzclaw WD, Kang MI, Kobayashi A, Yamamoto $M$, et al. Protection against electrophile and oxidant stress by induction of the phase 2 response: fate of cysteines of the Keap1 sensor modified by inducers. Proc Natl Acad Sci U S A 2004; 101: 2040-5.

29 Suttner DM, Dennery PA. Reversal of HO-1 related cytoprotection with increased expression is due to reactive iron. FASEB J 1999; 13: 1800-9.

30 Quesada A, Ogi J, Schultz J, Handforth A. C-terminal mechano-growth factor induces heme oxygenase-1-mediated neuroprotection of $\mathrm{SH}$ SY5Y cells via the protein kinase C/Nrf2 pathway. J Neurosci Res 2011; 89: 394-405.

31 Ross D, Kepa JK, Winski SL, Beall HD, Anwar A, Siegel D. NAD(P) $\mathrm{H}$ :quinone oxidoreductase 1 (NQO1): chemoprotection, bioactivation, gene regulation and genetic polymorphisms. Chem Biol Interact 2000; 129: 77-97.

32 Lin HY, Yeh WL, Huang BR, Lin C, Lai CH, Lin H, et al. Desipramine protects neuronal cell death and induces heme oxygenase-1 expression in Mes23.5 dopaminergic neurons. PLoS One 2012; 7: e50138.

33 Mineli A, Conte C, Grottelli S, Bellezza M, Cacciatore I, Bolanos JP. Cyclo(His-Pro) promotes cytoprotection by activating Nrf2-mediated up-regulation of antioxidant defence. J Cell Mol Med 2009; 13: 1149-61.

34 Jiang G, Hu Y, Liu L, Cai J, Peng C, Li Q. Gastrodin protects against $\mathrm{MPP}(+)$-induced oxidative stress by up regulates heme oxygenase-1 expression through $\mathrm{p} 38 \mathrm{MAPK} / \mathrm{Nrf2}$ pathway in human dopaminergic cells. Neurochem Int 2014; 75: 79-88.

35 Johnson JA, Johnson DA, Kraft AD, Calkins MJ, Jakel RJ, Vargas MR, et al. The NRF2/ARE pathway: an indicator and modulator of oxidative stress in neurodegeneration. Ann N Y Acad Sci 2008; 1147: 61-9.

36 Ye S, Chen M, Jiang Y, Chen M, Zhou T, Wang Y, et al. Polyhydroxylated fullerene attenuates oxidative stress-induced apoptosis via a fortifying Nrf2-regulated cellular antioxidant defence system. Int J Nanomedicine 2014; 9: 2073-87. 